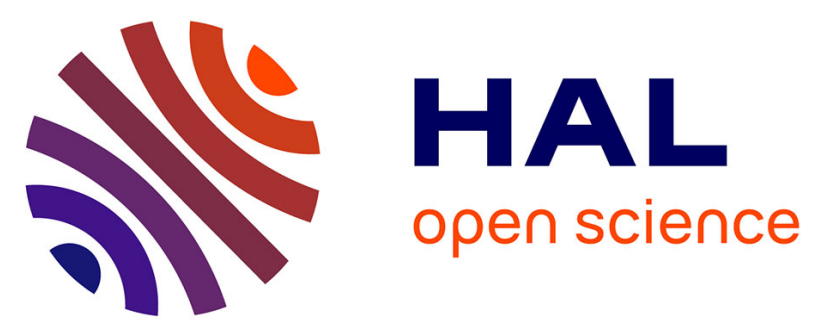

\title{
Impact of Synchronization Errors on the Performance of ACO-OFDMA Signaling for Medical Extra-WBAN Links
}

\author{
Md Jahid Hasan, Mohammad Ali Khalighi, Luis Nero Alves, Bastien \\ Béchadergue
}

\section{To cite this version:}

Md Jahid Hasan, Mohammad Ali Khalighi, Luis Nero Alves, Bastien Béchadergue. Impact of Synchronization Errors on the Performance of ACO-OFDMA Signaling for Medical Extra-WBAN Links. 2021 IEEE 32nd Annual International Symposium on Personal, Indoor and Mobile Radio Communications (PIMRC), 2021, Helsinki, Finland. 10.1109/PIMRC50174.2021.9569579 . hal-03452593

\section{HAL Id: hal-03452593 https://hal.science/hal-03452593}

Submitted on 30 Nov 2021

HAL is a multi-disciplinary open access archive for the deposit and dissemination of scientific research documents, whether they are published or not. The documents may come from teaching and research institutions in France or abroad, or from public or private research centers.
L'archive ouverte pluridisciplinaire HAL, est destinée au dépôt et à la diffusion de documents scientifiques de niveau recherche, publiés ou non, émanant des établissements d'enseignement et de recherche français ou étrangers, des laboratoires publics ou privés. 


\section{Impact of Synchronization Errors on the Performance of ACO-OFDMA Signaling for Medical Extra-WBAN Links}

\author{
$1^{\text {st }}$ Md Jahid Hasan \\ Aix-Marseille University, \\ CNRS, Centrale Marseille, \\ Institut Fresnel \\ Marseille, France \\ Oledcomm SAS, France \\ $2^{\text {nd }}$ Mohammad Ali Khalighi \\ Aix-Marseille University, \\ CNRS, Centrale Marseille, \\ Institut Fresnel \\ Marseille, France \\ ali.khalighi@fresnel.fr
}

jahid.hasan@fresnel.fr

\author{
$3^{\text {rd }}$ Luis Nero Alves \\ Instituto de Telecomunicações \\ Departamento de Electrónica, \\ Telecomunicações e Informática \\ Universidade de Aveiro \\ 3810-193 Aveiro, Portugal \\ nero@ua.pt
}

\author{
$4^{\text {th }}$ Bastien Béchadergue \\ Laboratoire d'Ingénierie des- \\ Systèmes de Versailles \\ Université de Versailles- \\ Saint-Quentin \\ Vélizy-Villacoublay, France \\ bastien.bechadergue@uvsq.fr
}

\begin{abstract}
This paper considers the use of infrared-based optical wireless communications for multi-user uplink wireless body-area networks. We propose the use of optical-orthogonal frequency division multiple access (O-OFDMA) signaling to manage the multiple access (MA) requirement for relatively high-rate medical applications. In particular, we consider asymmetrically clipped O-OFDMA and analyze its performance in terms of bit-errorrate and outage probability in the presence of multi-user time synchronization errors. These latter may occur due to mobility and random transmitter orientations, and will impact the link performance by inducing MA interference (MAI). We show that the effect of MAI increases with increasing the data rate. For instance, for 1 and $2 \mathrm{Mbps}$ data rates, to achieve a target biterror-rate of $10^{-3}$, the link distance is limited to 1.9 and $1 \mathrm{~m}$, respectively, compared to 2.2 and $1.8 \mathrm{~m}$ in the absence of MAI.

Index Terms-Wireless body area networks, optical-orthogonal frequency-division multiple access, synchronization errors, optical wireless communications.
\end{abstract}

\section{INTRODUCTION}

Real-time continuous remote monitoring of patients in hospitals gained particular attention during the COVID-19 pandemic due to the possibility of detecting patients' health conditions at an early stage and minimizing direct contact with them. Such a monitoring system may use on-body sensor nodes and a coordinator node $(\mathrm{CN})$ to collect and transmit medical data to a remote location through the use of a wireless body area network (WBAN) [1], [2]. At first, using intra-WBAN links, medical data from different sensors are transferred to the $\mathrm{CN}$ placed on the patient's body [3]. Then, these collected data are transmitted from the $\mathrm{CN}$ to an access point (AP) via an extraWBAN link [4]. Optical wireless communications (OWC) have recently emerged as a potential alternative to radio-frequency (RF) communications for medical WBANs [5], mainly due to their immunity to RF interference and the availability of a license-free unregulated spectrum [6]. Moreover, the inherent security features of OWC prevent the interception and hacking of confidential health information.
We focus in this paper on extra-WBAN uplink data transmission. In a realistic hospital ward scenario, several patients transmit their health data simultaneously, which should be managed by employing an efficient multiple access (MA) scheme. In optical WBANs, time- and code-division MA (TDMA and CDMA) are the most popular schemes proposed so far for relatively low data-rate $(\lesssim 100 \mathrm{Kbps})$ medical applications [7][9]. However, with increased data-rate, e.g., for specific medical applications such as Electromyography and capsule Endoscopy, complexity increases due to short time slots in TDMA and the required long signatures for CDMA. Therefore, to manage MA interference (MAI) efficiently, we propose here the use of optical-orthogonal frequency-division MA (O-OFDMA).

O-OFDMA is a multi-user extension of the popular OOFDM modulation scheme [10]. The interest in O-OFDM is that, in contrast to the classical optical on-off-keying, it is more energy-efficient, and resilient to channel frequency selectivity [11]. Here, we consider the use of an light-emittingdiode (LED) at the transmitter (Tx), where due to intensity modulation, the "time-domain" (TD) signal must be real and positive. Therefore, Hermitian symmetry (HS) is imposed on the "frequency-domain" (FD) signal to obtain a real-valued TD signal. To ensure positivity, either a DC bias is added to the TD signal, followed by zero-level clipping, resulting in the so called DCO-OFDM scheme, or by modulating the odd subcarriers followed by zero-level clipping, resulting in asymmetricallyclipped (AC)O-OFDM scheme [12], [13].

One major concern for uplink O-OFDMA is time synchronization. Indeed, users must be strictly time-synchronized regardless of their positions, i.e., the signals of the different users must arrive at the receiver $(\mathrm{Rx})$ at the same time. This timing synchronization is achieved based on the channel state information (CSI) which is estimated based on transmitting training sequences [14], [15]. However, as we will explain later in Section II, CSI may become outdated due to mobility and random Tx orientations, resulting ultimately in multi-user 
synchronization errors and thus MAI.

To the best of our knowledge, the effect of MAI on OOFDMA due to multi-user timing synchronization errors has never been investigated so far, which is the aim of this paper by considering the ACO-OFDMA scheme.

The remainder of the paper is organized as follows. In Section II, we present the system model description and assumptions on the hospital ward scenario. Next, Sections III and IV present the ACO-OFDMA scheme, and its performance analysis, respectively. Lastly, the main conclusions are drawn in Section V.

\section{SySTEM DESCRIPTION}

\section{A. General Assumptions}

Consider a hospital ward scenario with up to four patients. For extra-WBAN IR uplink transmission, the collected medical data from the $\mathrm{CN}$ placed on the patient's shoulder (to maximize the probability of line-of-sight, LOS, existence) are transmitted to an AP placed on the ceiling. Visible-light (VL) communication can be used for downlink transmission, as well as for multiuser synchronization. We consider a single AP covering an area of radius $r_{c e l l}$, see Fig. 1. A PIN photo-detector (PD) with no optical concentrator is used at the AP, ensuring a relatively large field-of-view (FOV). We assume that the data-rate requirement of each $\mathrm{CN}$ is on the order of Mbps [1].

Due to the distinct time and frequency offsets between mobile users, uplink synchronization in OFDMA is more difficult than in the downlink. Note that, due to the inherent absence of a carrier as in RF [15], we are only concerned by timing synchronization. As mentioned previously, timing offsets are estimated based on the CSI of each user. At first, in the uplink, each user transmits its training symbols at the beginning of each frame, based on which, the AP estimates the timing reference and then transmits the corrected timing information via the downlink. Note that, reciprocity between VL-uplink and IR-downlink does not hold for estimating CSI due to the mobility and different corresponding signal-to-noise ratios (SNRs) [16]. However, because of the round trip (i.e., uplink and downlink) propagation delay, residual timing errors may still be present for the uplink transmission at the AP. However, this synchronization problem can be avoided by taking into account two way propagation delay in fixing the cyclic prefix (CP) length [17]. Also, note that user mobility and random Tx orientations should be considered as introducing slow channel fading with a typical average coherence time of $100 \mathrm{~ms}$. This should result in negligible synchronization errors, given the frame rates on the order of $\mu$ s [18].

Nevertheless, medical sensors collect data periodically at different rates, with sensing intervals between 100 and $250 \mathrm{~ms}$, typically [19]. Therefore, the interval between the transmission of data frames from users can easily exceed the channel coherence time, resulting in outdated CSI, which can cause symbol timing offset when used for uplink synchronization. This can destroy the orthogonality between users, leading to MAI. In the sequel, for the sake of simplicity, we consider a

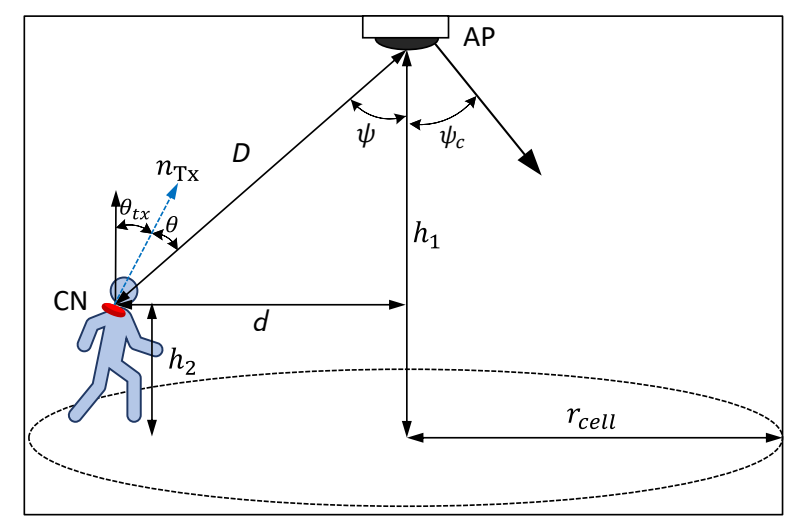

Fig. 1: Illustration of a LOS link configuration between a $\mathrm{CN}$ and the AP. $n_{\mathrm{Tx}}$ represents the normal vector corresponding to the Tx.

maximum of one symbol timing offset of interfering users with respect to a desired user.

\section{B. Channel Model}

For the sake of simulation simplicity, we consider here only the LOS link in our simulations and assume a Lambertian source model for the LED at the Tx (i.e., the CN). Denoting the channel attenuation by $H_{\mathrm{LOS}}[20]$,

$$
H_{\mathrm{LOS}}= \begin{cases}\frac{A_{\mathrm{PD}}(m+1)}{2 \pi D^{2}} \cos ^{m}(\theta) \cos (\psi) & ; 0 \leq \psi \leq \psi_{c} \\ 0 & ; \psi>\psi_{c}\end{cases}
$$

where $A_{\mathrm{PD}}$ stands for the PD's active area, $D$ is the link distance, $\psi$ denotes the incident angle at the AP, $\psi_{c}$ is the FOV of the Rx, $m$ is the LED Labmertian order, and $\theta$ refers to the $\mathrm{Tx}$ beam radiance angle that relies on the Tx orientation angle $\theta_{t x}$.

Considering the PIN PD responsivity $\mathcal{R}$ and the transmit optical power $P_{T \text { (opt) }}$, the received photo-current is given by $I_{r}=\mathcal{R} H_{\mathrm{LOS}} P_{T(\mathrm{opt})}$. Also, power spectral density corresponding to the one-sided background and thermal noises is [21]:

$$
N_{0} \approx 4 K_{B} T_{r} / R_{L}+2 q_{e} I_{b},
$$

where $K_{B}$ stands for the Boltzmann's constant, $R_{L}$ is the load resistance of the trans-impedance amplifier (TIA), $T_{r}$ denotes the $\mathrm{Rx}$ equivalent temperature, $q_{e}$ is the electron charge, and $I_{b}$ refers to the background current noise.

\section{DESCRIPTION OF THE ACO-OFDMA SCHEME}

Figure 2 shows the block diagram of ACO-OFDMA signaling. First, input bits from different sensors are grouped together and mapped by $M$-QAM constellation into complex symbols $\hat{X}_{\hat{K}}, \hat{K}=0,1, \cdots, \mathcal{N}-1$, where $\mathcal{N}$ denotes the number of subcarriers per user. The total number of subcarriers $\mathcal{N}_{s m}=\mathcal{N} \mathcal{T}$, where $\mathcal{T}$ refers to the maximum number of users. Denote by $X_{K}$ the symbols after subcarrier mapping. Since by ACOOFDMA only odd subcarriers are modulated [12], denoting the symbols after applying HS by $\breve{X}_{\breve{K}}, \breve{K}=0,1, \cdots, N-1$, 


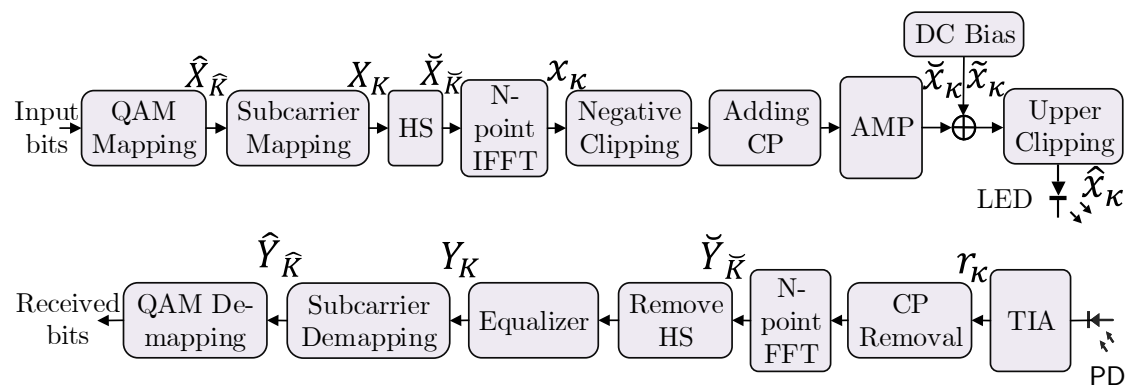

Fig. 2: Block diagram of the ACO-OFDMA signaling scheme.

we have $N=4 \mathcal{N}_{s m}+2$. Then, after $N$-point inverse FastFourier-transform (IFFT), the resulting TD signal $x_{\kappa}$ is [12]:

$$
x_{\kappa}=\frac{1}{\sqrt{N}} \sum_{\breve{K}=0}^{N-1} \breve{X}_{\breve{K}} \exp \left(j \frac{2 \pi \kappa \breve{K}}{N}\right) ; \kappa=0,1, \ldots, N-1 .
$$

Next, after negative clipping, at the end of each frame, a CP of length $N_{\mathrm{CP}}$ is inserted, before adding a DC bias $B_{\mathrm{DC}}$ to the amplified analog signal $\breve{x}_{\kappa}$ to obtain $\tilde{x}_{\kappa}$ :

$$
\tilde{x}_{\kappa}=\breve{x}_{\kappa}+B_{\mathrm{DC}} \text {. }
$$

Note that, $B_{\mathrm{DC}}$ is usually fixed by taking into account the LED dynamic range [11]. Finally, after upper clipping the signal $\tilde{x}_{\kappa}$, the resulting signal $\hat{x}_{\kappa}$ drives the LED.

At the Rx, as shown in Fig. 2, after removing DC bias, the CP is removed from the amplified signal frames, and an $N$-point FFT is performed to obtain the output signal [12]:

$$
\breve{Y}_{\breve{K}}=\frac{1}{\sqrt{N}} \sum_{\kappa=0}^{N-1} r_{\kappa} \exp \left(-j \frac{2 \pi \kappa \breve{K}}{N}\right) ; \breve{K}=0,1, \ldots, N-1
$$

Afterwards, HS is removed from $\breve{Y}_{\breve{K}}$, which is then passed through a single-tap equalization block to obtain $Y_{K}$. Then, after subcarrier demapping and QAM demodulation, the transmitted bits are recovered.

\section{Performance Analysis}

We present here a set of numerical results to study the performance of ACO-OFDMA for medical extra-WBAN uplink in the presence and absence of synchronization errors. Given the number of patients $\mathcal{T}=4$, we consider the number of symbols per patient $\mathcal{N}=16$ with the total number of mapped subcarriers $\mathcal{N}_{s m}=64$ and $N=258$ after imposing HS. For this configuration, considering a fixed transmission bandwidth $\mathcal{B}=8.25 \mathrm{MHz}$, we analyze the performance for $R_{b}=$ $1 \mathrm{Mbps}$ and $2 \mathrm{Mbps}$, corresponding to spectral efficiencies $\eta \approx 0.125$ and $0.25 \mathrm{bps} / \mathrm{Hz} / \mathrm{user}$, using 4-QAM and 16-QAM constellations, respectively. A low-power IR LED (Stanley, HDN1102W-TR) is considered [22], where I-V characteristics is approximated as linear. Given the LED turn-ON voltage, we set $B_{\mathrm{DC}}$ to $1.30 \mathrm{~V}$ and further consider upper clipping at

\begin{tabular}{|c|c|c|}
\hline Parameter & Symbol & Value \\
\hline Max. number of patients & $\mathcal{T}$ & 4 \\
\hline Transmission bandwidth & $\mathcal{B}$ & $8.25 \mathrm{MHz}$ \\
\hline Threshold BER & $\mathrm{BER}_{\mathrm{TH}}$ & $10^{-3}$ \\
\hline Cell Radius & $r_{\text {cell }}$ & $3 \mathrm{~m}$ \\
\hline AP height & $h_{1}$ & $3 \mathrm{~m}$ \\
\hline $\mathrm{CN}$ height & $h_{2}$ & $0.4 \mathrm{~m}$ \\
\hline Tx orientation angle & $\theta_{t x}$ & $-60^{\circ}-60^{\circ}$ \\
\hline LED wavelength [22] & - & $850 \mathrm{~nm}$ \\
\hline LED Lambertian order [22] & $m$ & 1 \\
\hline Power conversion efficiency [22] & $v$ & 0.8 \\
\hline PD responsivity & $\mathcal{R}$ & $0.6 \mathrm{~A} / \mathrm{W}$ \\
\hline PD active area & $A_{\mathrm{PD}}$ & $1 \mathrm{~cm}^{2}$ \\
\hline TIA load resistance & $R_{L}$ & $50 \Omega$ \\
\hline Rx noise temperature & $T_{r}$ & $300 \mathrm{~K}$ \\
\hline Rx FOV & $\psi_{c}$ & $70^{\circ}$ \\
\hline Ambient current noise [23] & $I_{b}$ & $1000 \mu \mathrm{A}$ \\
\hline Total mapped subcarriers & $\mathcal{N}_{\text {sm }}$ & 64 \\
\hline Subcarriers allocated per user & $\mathcal{N}$ & 16 \\
\hline $\mathrm{CP}$ length & $N_{\mathrm{CP}}$ & 2 \\
\hline DC-bias & $B_{\mathrm{DC}}$ & $1.30 \mathrm{~V}$ \\
\hline Upper clipping & - & $2 \mathrm{~V}$ \\
\hline
\end{tabular}
$2 \mathrm{~V}$ to avoid LED overheating. The simulation parameters are
TABLE I: Parameters Used for Numerical Simulations.

summarized in Table I. We use interleaved subcarrier mapping in our simulations.

Considering the number $\mathcal{N}$ of symbols per user before subcarrier mapping and the $M-\mathrm{QAM}$ constellation, the spectral efficiency of ACO-OFDMA,

$$
\eta=\frac{\log _{2}(M) \mathcal{N}}{N+N_{\mathrm{CP}}} \quad(\mathrm{bps} / \mathrm{Hz} / \text { user }) .
$$

Denoting the transmission bandwidth by $\mathcal{B}$, the data-rate $R_{b}$ is then $R_{b}=\mathcal{B} \eta$. Considering the LED electrical-tooptical power efficiency $v$, we have, $P_{T \text { (opt) }}=v P_{T \text { (elec) }}$, where $P_{T \text { (elec) }}$ refers to the average electrical transmit power, $P_{T(\text { elec })}=E\left\{\hat{x}_{\kappa}^{2}\right\}$, with $E\{\cdot\}$ denoting the expected value. Also, the average transmit energy per bit is given by $E_{b(\mathrm{elec})}=$ $P_{T \text { (elec) }} / R_{b}$. 


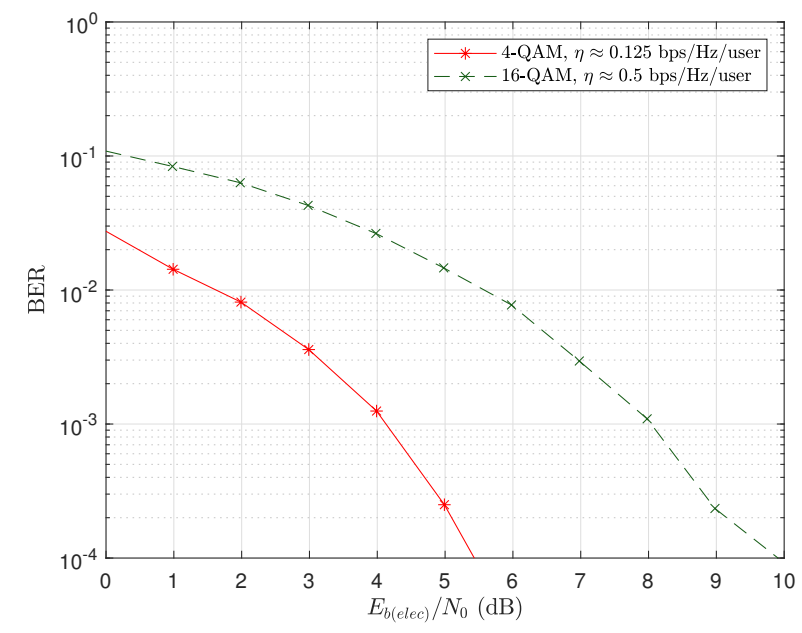

Fig. 3: BER performance comparison of ACO-OFDMA for data rates $R_{b}=$ $1 \mathrm{Mbps}$ and $2 \mathrm{Mbps}$. Patients are placed at the cell center. The electrical transmit power $P_{T(\text { elec })}$ is set to $35 \mathrm{~mW}$.

\section{A. BER Performance}

Let's first study the BER performance considering a perfect time-synchronous extra-WBAN uplink multi-user scenario. We assume that patients are positioned at the cell center, i.e., at $d=0 \mathrm{~m}$, and CNs are pointing straight to the ceiling. Figure 3 compares the BER performance for different $R_{b}$, for $P_{T(\text { elec })}=$ $35 \mathrm{~mW}$ (which allows to have no clipping noise, as we will show later). As expected, for $R_{b}=2 \mathrm{Mbps}$, a higher $E_{b} / N_{0}$ is required to achieve a target $\mathrm{BER}, \mathrm{BER}_{\mathrm{TH}}=10^{-3}$ due to the larger signal constellation used.

Now, we analyze the performance with respect to $P_{T \text { (elec) }}$ (set equal for all users), where we change the distance $d$ of the desired user from the cell center, whereas the other three users are kept at the cell center. BER plots are presented in Fig. 4 for $R_{b}=1 \mathrm{Mbps}$, where we notice that, as expected, due to lower received SNR with increasing $d$, the required $P_{T \text { (elec) }}$ to attain a target BER increases. For a too high transmit power, e.g., $P_{T \text { (elec) }} \gtrsim 41 \mathrm{~mW}$ for $d=3 \mathrm{~m}$, we notice a detrimental impact of clipping noise on the BER. Figure 5 presents similar results for $R_{b}=2 \mathrm{Mbps}$, using 16-QAM constellation. We notice a higher SNR requirement as the signal constellation size is larger, and also, a more noticeable clipping noise effect, which is due to the increased signal peak-to-average-power ratio.

\section{B. Effect of Synchronization Errors}

Here, we investigate the impact of multi-user uplink synchronization errors due to outdated CSI of the interferers on the desired user performance. For the sake of simulation simplicity, we assume that the interferers have a maximum offset of one symbol duration with respect to the desired user, which is, in turn, perfectly phase-synchronized with the Rx sampling clock.

1) Fixed Tx Orientation: At first, we assume that the $\mathrm{CN}$ of the desired user is pointed straight towards the ceiling,

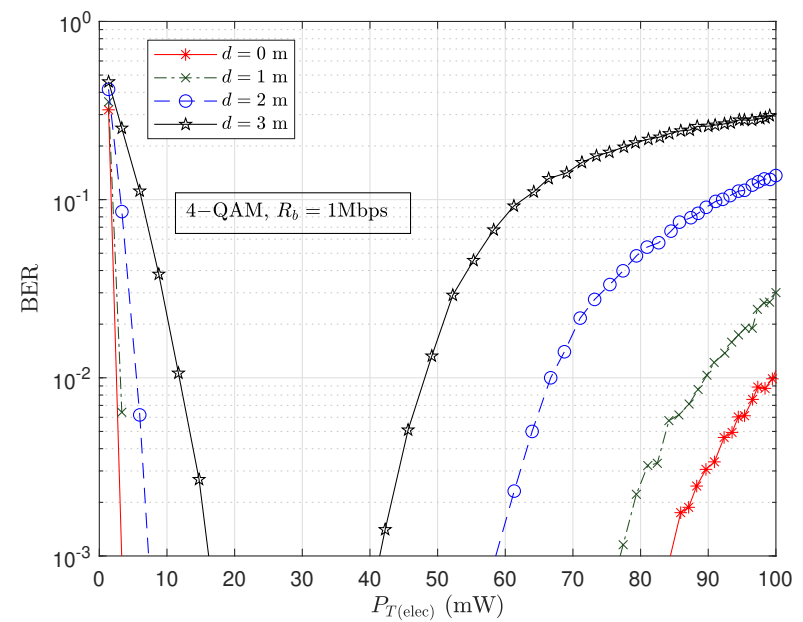

Fig. 4: BER performance of ACO-OFDMA for $R_{b}=1 \mathrm{Mbps}$ for different distances $d$ of the desired patient with respect to the electrical transmit power $P_{T \text { (elec) }}$. The three other patients are placed at the cell center.

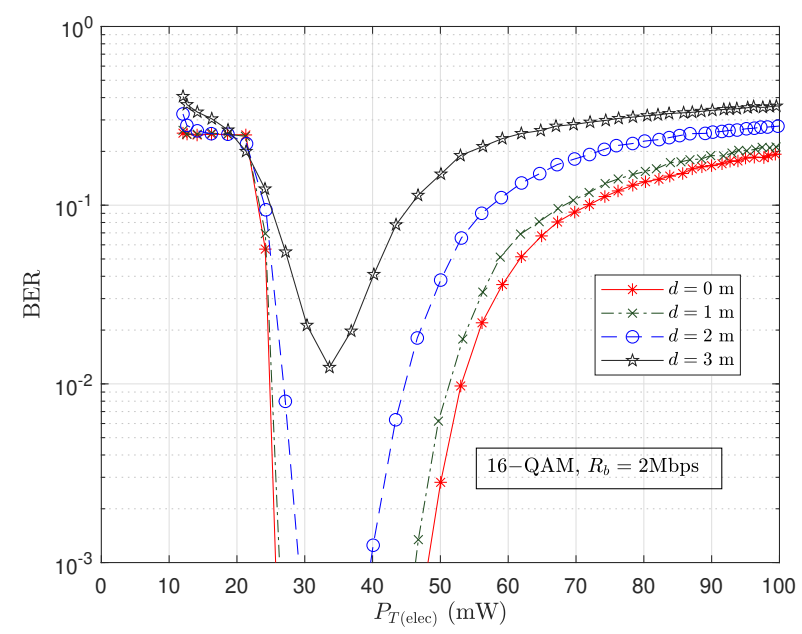

Fig. 5: BER performance of ACO-OFDMA for $R_{b}=2 \mathrm{Mbps}$ for different distances $d$ of the desired patient with respect to the electrical transmit power $P_{T(\text { elec })}$. The three other patients are placed at the cell center.

i.e., $\theta_{t x}=0^{\circ}$, regardless of its position. The interferers are considered to be placed at the cell center, where their CNs are randomly oriented based on Gaussian distributed random $\mathrm{Tx}$ orientations $\theta_{t x}$ in the interval of $\left(-60^{\circ}, 60^{\circ}\right)$. Based on this, we have used $10^{6}$ randomly generated $\theta_{t x}$ in our simulations.

Figure 6 shows the effect of MAI resulting from synchronization errors on the BER of the desired user, with distance $0 \leq d \leq 3 \mathrm{~m}$ from the cell center. As expected, the MAI effect increases by increasing $d$. As reference, we have also shown the BER plots for the no-MAI case, denoted by NMAI. Also, the impact of MAI is more detrimental for $R_{b}=2 \mathrm{Mbps}$ due to the larger signal constellation used.

2) Random Tx Orientation: Now, we take into account random Tx orientations for the desired user in addition to the 


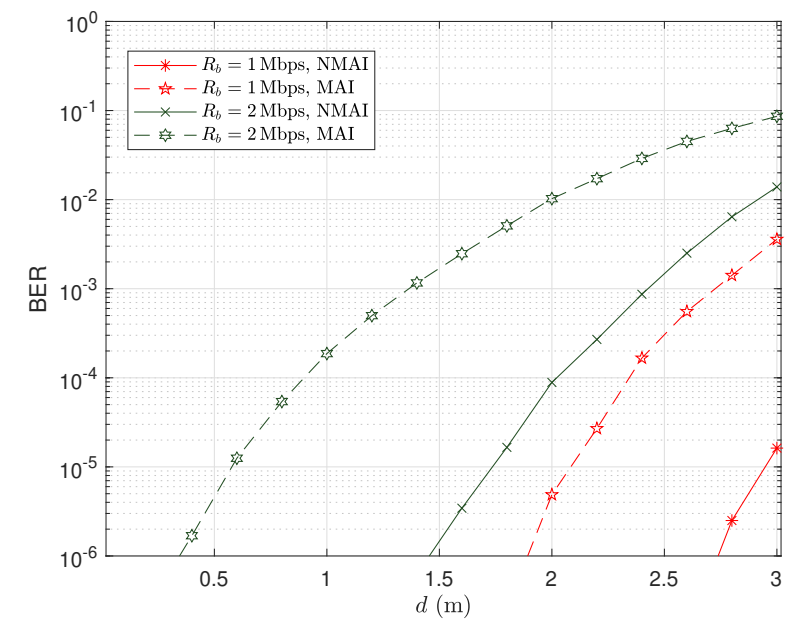

Fig. 6: BER performance versus distance $d$ of the desired user from the cell center for $R_{b}=1$ and $2 \mathrm{Mbps}$. MAI represents the case where three other users introduce multi-user time-synchronization errors with the desired user. NMAI refers to no-MAI.

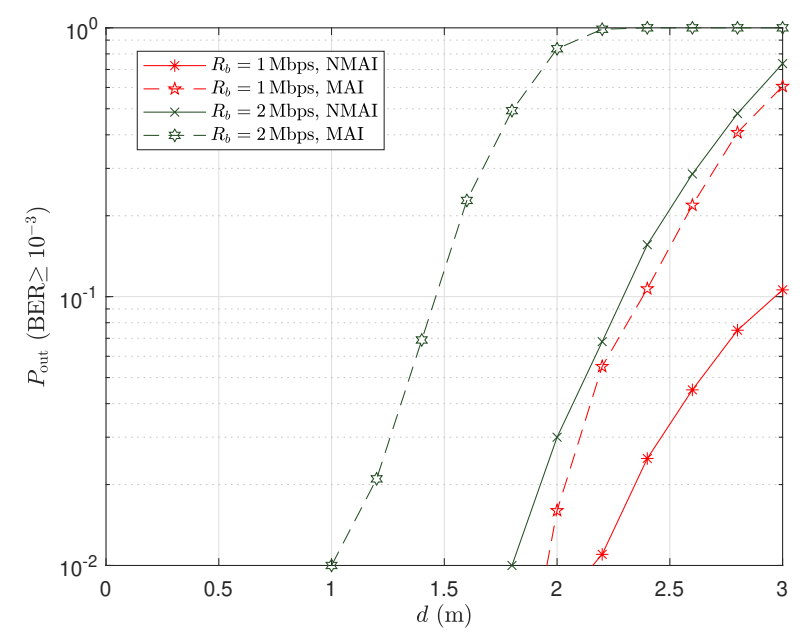

Fig. 7: Outage probability versus the distance $d$ of the desired user from the cell center for $R_{b}=1 \mathrm{Mbps}$ and $2 \mathrm{Mbps}$ under random Tx orientations for all users (including the desired user).

interferers for the same scenario as in the previous paragraph. Due to the random nature of the desired user's channel, we consider the performance in terms of outage probability $P_{\text {out }}$, which is defined as the probability that the BER exceeds a threshold, here, $\mathrm{BER}_{\mathrm{TH}}=10^{-3}$. The $P_{\text {out }}$ plots are presented in Fig. 7 for different distances $d$ of the desired user. As expected, $P_{\text {out }}$ increases as the desired user moves from the cell center to the cell edge due to a higher probability of loosing the LOS link. Also, MAI increases with increased $d$. Note that, for $R_{b}=1 \mathrm{Mbps}$, the required quality of service $P_{\text {out }}=10^{-2}$ [8] is achieved for $d \lesssim 1.9 \mathrm{~m}$. For increased data-rate, and hence, signal constellation size, synchronization errors show a more penalizing effect.

\section{CONCLUSIONS}

We investigated the performance of ACO-OFDMA based uplink extra-WBAN signal transmission for multi-user medical applications. Considering a low-power IR LED and taking into account its limited dynamic range, we analyzed BER and $P_{\text {out }}$ performances in a hospital ward scenario exposed to indirect sunlight and demonstrated the detrimental impact of MAI resulting from time synchronization errors. In particular, for relatively high data rates, e.g., 2 Mbps using 16-QAM modulation, we showed a more penalizing effect of MAI, failing to achieve the target BER of $10^{-3}$ for distances larger than $1 \mathrm{~m}$. Future work will consider the effect of users mobility and efficient MAC-layer design to increase robustness against synchronization errors.

\section{ACKNOWLEDGMENT}

This work is supported by the European Union's Horizon 2020 research and innovation programme under the Marie SklodowskaCurie grant agreement no 764461 (VisIoN). It is also based upon work from COST Action CA19111 NEWFOCUS, supported by COST (European Cooperation in Science and Technology).

\section{REFERENCES}

[1] O. Haddad and M. A. Khalighi, "Enabling communication technologies for medical wireless body-area networks," in Global LiFi Congress (GLC), June 2019, pp. 1-5, Paris, France.

[2] S. Movassaghi, M. Abolhasan, J. Lipman, D. Smith, and A. Jamalipour, "Wireless body area networks: A survey," IEEE Communications Surveys \& Tutorials, vol. 16, no. 3, pp. 1658-1686, Mar. 2014.

[3] O. Haddad, M. A. Khalighi, S. Zvanovec, and M. Adel, "Performance analysis of optical intra-WBAN links," in International Symposium on Communication Systems, Networks and Digital Signal Processing (CSNDSP), July 2020, pp. 1-6, Porto, Portugal.

[4] O. Haddad, M. A. Khalighi, and S. Zvanovec, "Channel characterization for optical extra-WBAN links considering local and global user mobility," in Broadband Access Communication Technologies XIV, B. B. Dingel, K. Tsukamoto, and S. Mikroulis, Eds., vol. 11307, International Society for Optics and Photonics. SPIE, 2020, pp. 89 - 97.

[5] O. Haddad, M. A. Khalighi, S. Zvanovec, and M. Adel, "Channel characterization and modeling for optical wireless body-area networks," IEEE Open Journal of the Communications Society, vol. 1, pp. 760-776, June 2020.

[6] Z. Ghassemlooy, L. N. Alves, S. Zvanovec, and M. A. Khalighi, Eds., Visible Light Communications: Theory and Applications. CRC-Press, 2017.

[7] G. Sun, K. Wang, H. Yu, X. Du, and M. Guizani, "Priority-based medium access control for wireless body area networks with high-performance design," IEEE Internet of Things Journal, vol. 6, no. 3, pp. 5363-5375, June 2019.

[8] M. J. Hasan, M. A. Khalighi, J. García-Márquez, and B. Béchadergue, "Performance analysis of optical-CDMA for uplink transmission in medical extra-WBANs," IEEE Access, vol. 8, pp. 171 672-171685, Sept. 2020.

[9] M. J. Hasan, M. A. Khalighi, and B. Béchadergue, "Experimental implementation of Optical-CDMA for medical Extra-WBAN links," in 12th International Symposium on Communication Systems, Networks and Digital Signal Processing (CSNDSP), July 2020, Porto, Portugal.

[10] S. S. Bawazir, P. C. Sofotasios, S. Muhaidat, Y. Al-Hammadi, and G. K. Karagiannidis, "Multiple access for visible light communications: Research challenges and future trends," IEEE Access, vol. 6, pp. $26167-$ 26 174, May 2018.

[11] T. Essalih, M. A. Khalighi, S. Hranilovic, and H. Akhouayri, "Optical OFDM for SiPM-based underwater optical wireless communication links," Sensors, vol. 20, no. 21, Oct. 2020. 
[12] J. Armstrong and B. J. C. Schmidt, "Comparison of asymmetrically clipped optical OFDM and DC-biased optical OFDM in AWGN," IEEE Communications Letters, vol. 12, no. 5, pp. 343-345, May. 2008.

[13] Y. Sun, F. Yang, and L. Cheng, "An overview of OFDM-based visible light communication systems from the perspective of energy efficiency versus spectral efficiency," IEEE Access, vol. 6, pp. 60 824-60833, Oct. 2018.

[14] M. Morelli, L. Sanguinetti, and H. V. Poor, "A robust ranging scheme for OFDMA-based networks," IEEE Transactions on Communications, vol. 57, no. 8, pp. 2441-2452, Aug. 2009.

[15] Y. Jiang, Y. Wang, P. Cao, M. Safari, J. Thompson, and H. Haas, "Robust and low-complexity timing synchronization for DCO-OFDM LiFi systems," IEEE Journal on Selected Areas in Communications, vol. 36, no. 1, pp. 53-65, Jan. 2018.

[16] H. Marshoud, P. C. Sofotasios, S. Muhaidat, G. K. Karagiannidis, and B. S. Sharif, "On the performance of visible light communication systems with non-orthogonal multiple access," IEEE Transactions on Wireless Communications, vol. 16, no. 10, pp. 6350-6364, July 2017.

[17] M. Morelli, C.-C. J. Kuo, and M.-O. Pun, "Synchronization techniques for orthogonal frequency division multiple access (OFDMA): A tutorial review," Proceedings of the IEEE, vol. 95, no. 7, pp. 1394-1427, July 2007.

[18] A. A. Purwita, M. D. Soltani, M. Safari, and H. Haas, "Terminal orientation in OFDM-based LiFi systems," IEEE Transactions on Wireless Communications, vol. 18, no. 8, pp. 4003-4016, June 2019.

[19] M. Shu, D. Yuan, C. Zhang, Y. Wang, and C. Chen, "A MAC protocol for medical monitoring applications of wireless body area networks," Sensors, vol. 15, no. 6, pp. 12906-12931, June 2015.

[20] J. M. Kahn and J. R. Barry, "Wireless infrared communications," Proceedings of the IEEE, vol. 85, no. 2, pp. 265-298, Feb. 1997.

[21] F. Xu, M. Khalighi, and S. Bourennane, "Impact of different noise sources on the performance of PIN- and APD-based FSO receivers," in International Conference on Telecommunications (ConTel), June 2011, pp. 211-218, Graz, Austria.

[22] HDN1102W-TR Standard Product Specifications, Stanley Electric Co. Ltd., 2014.

[23] A. J. C. Moreira, R. T. Valadas, A. M. De, and O. Duarte, "Optical interference produced by artificial light," pp. 131-140, May 1997. 